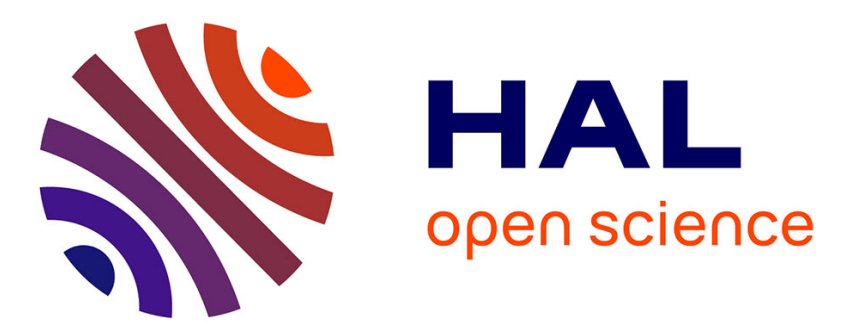

\title{
An exact solution for inflated orthotropic membrane tubes
}

Jean-Christophe Thomas, Anh Le Van

\section{To cite this version:}

Jean-Christophe Thomas, Anh Le Van. An exact solution for inflated orthotropic membrane tubes. Thin-Walled Structures, 2013, 67, pp.116-120. 10.1016/j.tws.2013.01.012 . hal-01005232

\section{HAL Id: hal-01005232 \\ https://hal.science/hal-01005232}

Submitted on 15 Oct 2016

HAL is a multi-disciplinary open access archive for the deposit and dissemination of scientific research documents, whether they are published or not. The documents may come from teaching and research institutions in France or abroad, or from public or private research centers.
L'archive ouverte pluridisciplinaire HAL, est destinée au dépôt et à la diffusion de documents scientifiques de niveau recherche, publiés ou non, émanant des établissements d'enseignement et de recherche français ou étrangers, des laboratoires publics ou privés.

\section{(c)(1)}

Distributed under a Creative Commons Attribution| 4.0 International License 


\title{
An exact solution for inflated orthotropic membrane tubes
}

\author{
Jean-Christophe Thomas*, Anh Le van

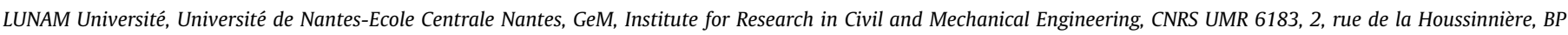
92208, 44322 Nantes Cedex 3, France

\begin{abstract}
Inflatable tubes must be pressurized before they are able to resist to external loadings. While there are many studies dedicated to the behavior of inflated tubes under bending or twisting, few results are available on the inflating stage when the beam is only submitted to the internal pressure. In this paper, an exact solution is proposed for a cylindrical tube made of an orthotropic membrane, with the orthotropy axes parallel to the circumferential and longitudinal directions of the cylinder, and undergoing finite deformations under internal pressure. The solution of the problem amounts to solving a cubic equation and analytical relations are given for the inflated geometry as well as the resultant stresses in the membrane. Numerical results are shown to compare very well with those obtained from a finite element code.
\end{abstract}

\section{Introduction}

Inflatable structures are membrane-like structures which acquire bearing capacities when submitted to an internal pressure. They possess highly interesting mechanical properties which account for their increasing development: they are light, easy to be folded, rapidly deployable, their storage volumes are small compared to standard structures and their manufacturing is less expensive.

Inflatable structures may be of various shapes; however, the geometry most investigated in the literature is the cylindrical tube as this is the component of many inflatable structures. Several studies have been carried out on inflatable tubes modeled as beams. The pioneering works were due to Comer and Levy [1] and Douglas [2] who provided analytical expressions for the deflection in an inflated cantilever beam assuming Bernoulli's kinematics. Later on, Webber [3] extended the results to beams subjected to bending and twisting. Main et al. [4] undertook experimental works and compared them with Comer and Levy's [1] analytical results. They also took into account the biaxial state of the fabric in [5] and were the first to investigate the dynamic response of inflatable beam structures made of orthotropic materials [6]. More recently, the behavior of inflatable tubes submitted to distributed loads in ocean aquaculture was investigated by Suhey et al. [7].

Other works have been conducted in order to better take account of the shear effect in inflatable beams. Steeves [9] proposed a theory based on the principle of minimal potential energy including the transverse shear deformation and gave the solution in terms of Green functions. Fichter [8] obtained a loaddeflection formula where the inflating pressure appears explicitly in the shear stiffness by assuming Timoshenko's kinematics. Adopting the same kinematics, Wielgosz and Thomas [10] proposed a theory dedicated to highly inflated panels, taking into account the deformed configuration and the internal pressure as a following force. The results were subsequently extended to highly inflated tubes in [11]. By reformulating Fichter's formulation with the virtual work principle, Le van and Wielgosz [12] obtained simple formulae involving the pressure both in the bending and shear stiffnesses. Their results were extended to the study of the dynamic behavior of inflated beams by Thomas et al. [13] and more recently to the case of orthotropic fabrics by Apedo et al. [14].

In fact, the study of an inflatable beam should be concerned with two successive stages: (i) the inflating stage when the beam is only submitted to the internal pressure and acquires its bearing capacities; (ii) the bending stage when the inflated beam is subjected to other external loadings. The above-mentioned studies were mainly focused on the second stage, assuming that the inflated state, which defines the reference configuration of the beam, is known. The reference radius and length, which vary as functions of the pressure, were computed from linear formulae in Le van and Wielgosz's paper [12], and also in Wang et al. analysis [15] of the wrinkling and collapse of inflated beams. These formulae are a good approximation in the linear context as long as the beam undergoes small deformations. However, one needs to use exact relations for the reference geometry in the case when the beam is submitted to high pressures giving rise to 
finite deformations. As it turns out, the precise knowledge of the reference geometry of the beam is a necessary condition for the accurate prediction of the subsequent bending response. This question was first dealt with by Douglass [2] on an inflated beam made of an incompressible isotropic hyperelastic material.

This paper focuses on the inflating stage of the beam and aims to an exact solution for an inflatable cylindrical tube made of an orthotropic membrane, with the orthotropy axes parallel to the circumferential and longitudinal directions of the cylinder. The analysis is carried out in the framework of finite deformations with a Saint-Venant Kirchhoff material. The solution of the problem will be shown to reduce to solving a cubic equation and analytical relations will be given for the final geometry as well as the resultant stresses in the membrane. In the last section of the paper, numerical computations will be made on different kinds of constitutive fabrics of the membrane and the results will be shown to compare very well with those obtained from a finite element code.

\section{Analytical solution for an inflated orthotropic thin tube}

The analytical solution for an inflated cylindrical tube made of a hyperelastic isotropic material and undergoing finite deformations can be found in [16, pp. 253-256]. Here, the analytical solution for an orthotropic tube, in the limit case of vanishing thickness, is established in the same spirit as in the isotropic case. The problem is formulated in finite deformations and one has to distinguish between the reference and the final configurations of the tube.

Consider a tube of inner radius $A$, outer radius $B$, thickness $H=B-A$ and length $L$ in the reference state, see Fig. 1 . The tube is closed with rigid end caps and submitted to an internal pressure $p$. In the final inflated state, the inner radius is denoted $a$, the outer radius $b$, the thickness $h=b-a$ and the length $\ell$. The tube is made of an orthotropic material, with the warp direction parallel to the tube axis and the weft direction parallel to the circumference.

We shall choose the cylindrical coordinate system, with basis vectors $\mathbf{e}_{r}, \mathbf{e}_{\theta}, \mathbf{e}_{z}$, to describe the axisymmetric problem. The position of any particle of the tube in the reference state is $\mathbf{X}=R \mathbf{e}_{r}+Z \mathbf{e}_{z}$, where $R$ is the reference radius and $Z$ the reference axial coordinate. The position of the same particle in the final state is $\mathbf{X}=r \mathbf{e}_{r}+z \mathbf{e}_{z}$. The deformation is defined by

$r=r(R), \quad z=\lambda_{z} Z$

where the function $r(R)$ and the constant $\lambda_{z}$ are to be determined. The deformation gradient tensor then is

$$
\begin{array}{r}
\mathbf{F}=\lambda_{r}(R) \mathbf{e}_{r} \otimes \mathbf{e}_{r}+\lambda_{\theta}(R) \mathbf{e}_{\theta} \otimes \mathbf{e}_{\theta}+\lambda_{z} \mathbf{e}_{z} \otimes \mathbf{e}_{z} \\
\text { where } \lambda_{r}(R)=\frac{d r}{d R} \quad \text { and } \quad \lambda_{\theta}(R)=\frac{r}{R}
\end{array}
$$

It is assumed that the tube is made of a hyperelastic orthotropic material obeying the Saint-Venant Kirchhoff law $\boldsymbol{\Sigma}=\mathbf{D}: \mathbf{E}$, relating the second Piola-Kirchhoff stress tensor $\boldsymbol{\Sigma}$ to the Green strain tensor $\mathbf{E}=\left(\mathbf{F}^{T} \mathbf{F}-\mathbf{I}\right) / 2$ via the elasticity tensor $\mathbf{D}$ (the symbol
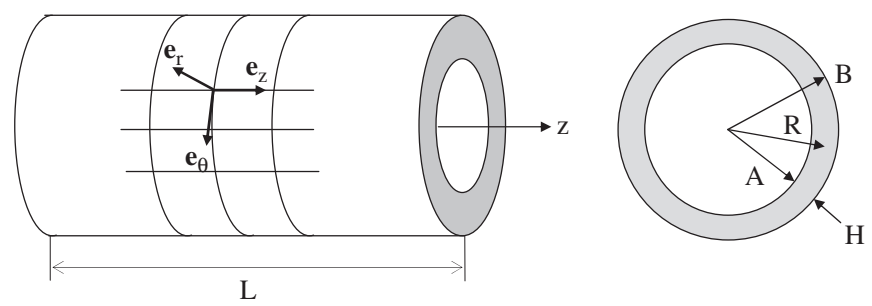

Fig. 1. Reference geometry of the tube.
' $\because$ ' denotes the double contraction between the fourth-order tensor $\mathbf{D}$ and the second-order tensor E). Young's moduli in the longitudinal (warp), transversal (weft) and normal directions are denoted $E_{\ell}, E_{t}$ and $E_{n}$, respectively; Poisson's ratios are $v_{i j}$, $i, j \in\{\ell, t, n\}$ satisfying $v_{i j} / E_{i}=v_{j i} / E_{j}$. Here, since the orthotropy directions are parallel to the vectors of the cylindrical basis, the second Piola-Kirchhoff stress tensor is

$\boldsymbol{\Sigma}=\Sigma_{r r} \mathbf{e}_{r} \otimes \mathbf{e}_{r}+\Sigma_{\theta \theta} \mathbf{e}_{\theta} \otimes \mathbf{e}_{\theta}+\Sigma_{z z} \mathbf{e}_{z} \otimes \mathbf{e}_{z}$

where the stress components are related to the strain components by

$\left\{\begin{array}{c}E_{r r} \\ E_{\theta \theta} \\ E_{z z}\end{array}\right\}=\frac{1}{2}\left\{\begin{array}{c}\lambda_{r}^{2}(R)-1 \\ \lambda_{\theta}^{2}(R)-1 \\ \lambda_{z}^{2}-1\end{array}\right\}=\left[\begin{array}{ccc}1 / E_{n} & -v_{t n} / E_{t} & -v_{\ell n} / E_{\ell} \\ -v_{n t} / E_{n} & 1 / E_{t} & -v_{\ell t} / E_{\ell} \\ -v_{n \ell} / E_{n} & -v_{t \ell} / E_{t} & 1 / E_{\ell}\end{array}\right]\left\{\begin{array}{c}\Sigma_{r r} \\ \Sigma_{\theta \theta} \\ \Sigma_{z z}\end{array}\right\}$

The Cauchy stress tensor can be derived using by $\boldsymbol{\sigma}=(1 / J) \mathbf{F} \boldsymbol{\Sigma} \mathbf{F}^{T}$, where $J=\operatorname{det} \mathbf{F}=\lambda_{r}(R) \lambda_{\theta}(R) \lambda_{z}$. It then follows that $\boldsymbol{\sigma}=\sigma_{r r} \mathbf{e}_{r} \otimes \mathbf{e}_{r}+\sigma_{\theta \theta} \mathbf{e}_{\theta} \otimes \mathbf{e}_{\theta}+\sigma_{z z} \mathbf{e}_{z} \otimes \mathbf{e}_{z}$, where

$$
\begin{gathered}
\sigma_{r r}(r)=\frac{\lambda_{r}(R)}{\lambda_{\theta}(R) \lambda_{z}} \Sigma_{r r}(R), \quad \sigma_{\theta \theta}(r)=\frac{\lambda_{\theta}(R)}{\lambda_{z} \lambda_{r}(R)} \Sigma_{\theta \theta}(R) \\
\sigma_{z z}(r)=\frac{\lambda_{z}}{\lambda_{\theta}(R) \lambda_{r}(R)} \Sigma_{z z}(R)
\end{gathered}
$$

Let us now assume that the tube is very thin, i.e. $H \ll A$, and accordingly make the following assumptions:

(i) The stretches $\lambda_{r}$ and $\lambda_{\theta}$ do not depend on $R$.

(ii) The radial stress is much less than the hoop and axial

$$
\text { stresses: } \Sigma_{r r} \ll \Sigma_{\theta \theta} \text { and } \Sigma_{z z} \text {. }
$$

Consider the relationship $|d \mathbf{X}| /|d \mathbf{X}|=\sqrt{\mathbf{N C N}}$, where $\mathbf{C}=\mathbf{F}^{T} \mathbf{F}$ is the right Cauchy deformation tensor, $d \mathbf{X}$ is a material line element and $d \mathbf{x}$ the spatial counterpart. Here, the cylindrical components of the deformation gradient tensor $\mathbf{F}$ are constant in view of Relation (2) and assumption (6). Consequently, Relation $d \mathbf{x}=\mathbf{F} d \mathbf{X}$ and then $|d \mathbf{x}| /|d \mathbf{X}|=\sqrt{\mathbf{N C N}}$ also hold for any vectors $d \mathbf{X}$ and $d \mathbf{x}$, not necessarily of infinitesimal lengths. Thus, the latter relationship can be applied to a point on the internal wall of the membrane, with $\mathbf{d X}=H \mathbf{e}_{r}, \mathbf{N}=\mathbf{e}_{r}$, then $\mathbf{d x}=h \mathbf{e}_{r}$, to give the stretch $\lambda_{r}$ in terms of the thicknesses

$\frac{h}{H}=\sqrt{\mathbf{e}_{r} \mathbf{C e}_{r}}=\lambda_{r}$

The final thickness-to-radius ratio $h / a$ can be recast as

$\frac{h}{a}=\frac{h}{H} \frac{H}{A} \frac{A}{a}=\frac{H}{A} \frac{\lambda_{r}}{\lambda_{\theta}}$

For the inflated membrane at hand, one should have $\lambda_{\theta}=a / A \geq 1$ and $0 \leq \lambda_{r}=h / H \leq 1$, ratio $\lambda_{r} / \lambda_{\theta}$ is then bounded and the assumption $H / A \ll 1$ leads to $h / a \ll 1$. On the other hand, taking into account assumption (7) in Relation (4) entails the constitutive law in plane stress conditions:

$\left\{\begin{array}{c}\Sigma_{\theta \theta} \\ \Sigma_{z z}\end{array}\right\}=\left[\begin{array}{cc}\bar{E}_{t} & v_{\ell t} \bar{E}_{t} \\ v_{t \ell} \bar{E}_{\ell} & \bar{E}_{\ell}\end{array}\right]\left\{\begin{array}{c}E_{\theta \theta} \\ E_{z z}\end{array}\right\}$

where $\bar{E}_{\ell}=E_{\ell} /\left(1-v_{t \ell} v_{\ell t}\right)$ and $\bar{E}_{t}=E_{t} /\left(1-v_{t \ell} v_{\ell t}\right)$.

From assumption (6) and Relation (10), the Piola-Kirchhoff stresses $\Sigma_{\theta \theta}$ and $\Sigma_{z z}$ do not depend on $R$. This, in turn, shows that the Cauchy stresses $\sigma_{\theta \theta}$ and $\sigma_{z z}$ in (5) $)_{b-c}$ are independent of $r$. The hoop stress $\sigma_{\theta \theta}$ is obtained by integrating the equilibrium equation $\sigma_{r r, r}+(1 / r)\left(\sigma_{r r}-\sigma_{\theta \theta}\right)=0$ and taking into account boundary conditions $\sigma_{r r}(r=a)=-p$ and $\sigma_{r r}(r=b)=0$ on the inner and 
outer walls:

$\sigma_{\theta \theta}=\frac{p a}{h}=\frac{p A}{H} \frac{\lambda_{\theta}}{\lambda_{r}}$

whereas the axial stress $\sigma_{z z}$ is derived from boundary condition $\int_{S(z=\ell)} \sigma_{z z} d S=p \pi a^{2}$ on the end cross-section $z=\ell$ and the above property $h / a \ll 1$ :

$\sigma_{z z}=p \frac{a^{2}}{b^{2}-a^{2}}=p \frac{a^{2}}{2 a h\left(1+\frac{h}{2 a}\right)} \approx \frac{p a}{2 h}=\frac{p A}{2 H} \frac{\lambda_{\theta}}{\lambda_{r}}$

Eventually, combining Eqs. (5) $b-c$ with (10)-(12) leads to a set of two equations with two unknowns $\lambda_{z}$ and $\lambda_{\theta}$ :

$\left\{\begin{array}{l}\lambda_{z}^{3}+\frac{2 v_{\ell t} p A}{E_{\ell}^{*}} \lambda_{z}^{2}-\frac{p A}{E_{\ell}^{*}} \lambda_{\theta}^{2}-\lambda_{z}=0 \\ \lambda_{\theta}^{2} \lambda_{z}-\frac{2 p A}{E_{t}^{*}} \lambda_{z}^{2}+\frac{p A v_{t \ell}}{E_{t}^{*}} \lambda_{\theta}^{2}-\lambda_{z}=0\end{array}\right.$

where $E_{t}^{*}=H E_{t}$ and $E_{\ell}^{*}=H E_{\ell}$ are the products of Young's moduli with the reference thickness of the membrane. Note that the thickness can hardly be measured in thin membranes and only the products $E_{t}^{*}$ and $E_{\ell}^{*}$ can be determined experimentally.

Let us extract $\lambda_{\theta}$ from (13)

$\lambda_{\theta}^{2}=\frac{E_{\ell}^{*}}{p A} \lambda_{z}^{3}+2 v_{\ell t} \lambda_{z}^{2}-\frac{E_{\ell}^{*}}{p A} \lambda_{z}$

Inserting this expression into $(13)_{b}$ then gives a cubic equation with unknown $\lambda_{z}$ :

$\lambda_{z}^{3}+\frac{3 p A v_{\ell t}}{E_{\ell}^{*}} \lambda_{z}^{2}-\left[1+\frac{2 p^{2} A^{2}}{E_{\ell}^{*} E_{t}^{*}}\left(1-v_{\ell t} v_{t \ell}\right)\right] \lambda_{z}-\frac{p A}{E_{\ell}^{*}}\left(1+v_{\ell t}\right)=0$

Eq. (15) of the form $\lambda_{z}^{3}+\beta \lambda_{z}^{2}+\gamma \lambda_{z}+\delta=0$ can be solved using Cardan's formula. The solution depends on the sign of the discriminant $\Delta=Q^{2}+4 P^{3} / 27$, where $P=\gamma-\beta^{2} / 3$ (to be distinguished from the internal pressure $p$ ) and $Q=2 \beta^{3} / 27-\beta \gamma / 3+\delta$. For all the usual numerical values of technical fabrics considered in this work, one finds $P<0$ and the discriminant $\Delta$ is negative. Consequently, use may be made of Viete's formulation to obtain an analytical expression for $\lambda_{z}$ :

$\lambda_{z}=2 \sqrt{\frac{-P}{3}} \cos \left(\frac{\varphi}{3}\right)-\frac{p A v_{\ell t}}{E_{\ell}^{*}} \quad$ with $\left.\varphi=\arccos \frac{3 Q}{2 P \sqrt{-P / 3}} \in\right]-\pi, \pi[$

The final radius and length of the membrane tube are then obtained by $a=\lambda_{\theta} A$ and $\ell=\lambda_{z} L$. The resultants of the Cauchy stresses on the thickness are given by $h \sigma_{\theta \theta}=2 h \sigma_{z z}=\lambda_{\theta} p A$. The thickness ratio $h / H$ cannot be determined as it involves material properties in the radial direction which are unknown.

It should be noted that in small deformations, $\lambda_{\theta} \approx 1$ and $\lambda_{z} \approx 1$, Relations (14) and (15) can be linearized to give

$a=A+\frac{p A^{2}}{2 E_{t}^{*}}\left(2-v_{t \ell}\right), \quad \ell=L+\frac{p A L}{2 E_{\ell}^{*}}\left(1-2 v_{\ell t}\right)$

These results are very close to Apedo's analytical solution [14].

\section{Numerical validation}

In this section, Relations (14) and (16) are applied on a tube of reference radius $A=0.15 \mathrm{~m}$, reference length $L=3 \mathrm{~m}$ and reference thickness $H=10^{-3} \mathrm{~m}$; and the results are compared to those obtained with finite element results just in order to verify the consistency of the analytical solution.

The tube is made of a thin fabric which is modeled as a membrane. Two typical coated fabrics are considered with the material properties summarized in Table 1. Fabric no. 1, similar to
Table 1

Material properties of the membranes.

\begin{tabular}{lcc}
\hline & Fabric no. 1 & Fabric no. 2 \\
\hline$E_{t}^{*}($ Pa m $)$ & 50000 & 50000 \\
$E_{\ell}^{*}($ Pa m $)$ & 50000 & 35000 \\
$G_{\ell t}^{*}($ Pa m $)$ & 12500 & 12500 \\
$v_{\ell t}$ & 0.08 & 0.3 \\
$v_{t \ell}$ & 0.08 & 0.21 \\
\hline
\end{tabular}

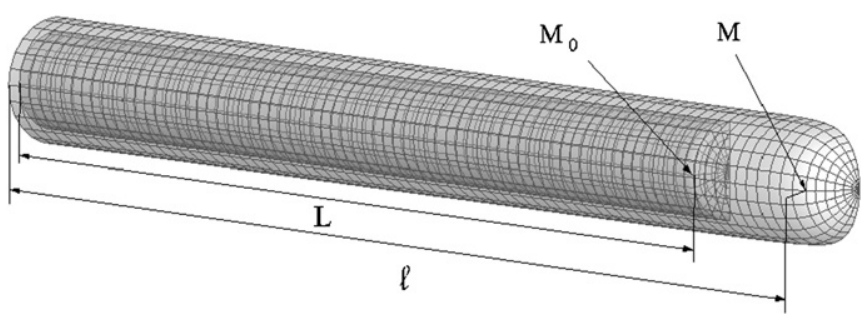

Fig. 2. A typical finite element mesh and the deformed shape after inflation.

that used in [14], is a balanced fabric with equal warp and weft moduli $E_{\ell}^{*}$ and $E_{t}^{*}$, whereas fabric no. 2 is unbalanced. The coefficient $G_{\ell t}^{*}=H G_{\ell t}$, equal to the product of the shear coefficient of the fabric with the reference thickness, is not involved in the analytical solution but is necessary for the finite element computations below.

All the analytical results obtained from Relations (14) and (15) are compared with finite element results provided by a general purpose in-house program dedicated to nonlinear structural mechanics problems. The finite element method is used to discretize the total Lagrangian formulation of the continuum problem and gives rise to a algebraic nonlinear equation system which can be solved by the Newton iterative scheme. The tangent stiffness matrix is the sum of the stiffness due to the internal forces and the stiffness due to the pressure which is a follower loading.

For the finite element computations, the tube is discretized into membrane elements with zero bending stiffness and the pressure $p$ is applied incrementally. At the first step when $p$ is zero, the stiffness matrix is singular and it is necessary to add a small fictitious pre-stress for the convergence. This pre-stress is immediately removed in the subsequent steps.

Due to the symmetry, only half of the beam has to be discretized. In the section $z=0$, the axial displacement of all nodes is prescribed to zero, in-plane displacements of some adequately chosen nodes are also set to zero in order to eliminate rigid-body motions, without preventing radial displacements. The mesh contains 3003 nodes and 943 six- or eight-node isoparametric membrane elements, see Fig. 2 . The chosen mesh proves to be sufficient for all numerical computations of interest since in the case of the fabric no. 1 submitted to pressure $p=10^{5} \mathrm{~Pa}$ for instance, the difference with a finer mesh containing 8183 nodes is only $0.068 \%$ on the length and $0.0015 \%$ on the radius.

Fig. 3 shows the change of geometry obtained with the finite element membrane computations as the pressure $p$ varies from $20 \mathrm{kPa}$ to $200 \mathrm{kPa}$. As can be seen in Fig. 2, the change in geometry may be significant so that the nonlinear computations are quite necessary. The final length $\ell$ of the inflated tube is obtained from the axial displacement of any particle belonging to the intersection between the cylindrical surface and the end cross-section (the initial and final positions, $M_{0}$ and $M$, of such a particle are shown in Fig. 2). The final radius $a$ is uniform over about $95 \%$ of the length and is measured at half-length of the tube. 


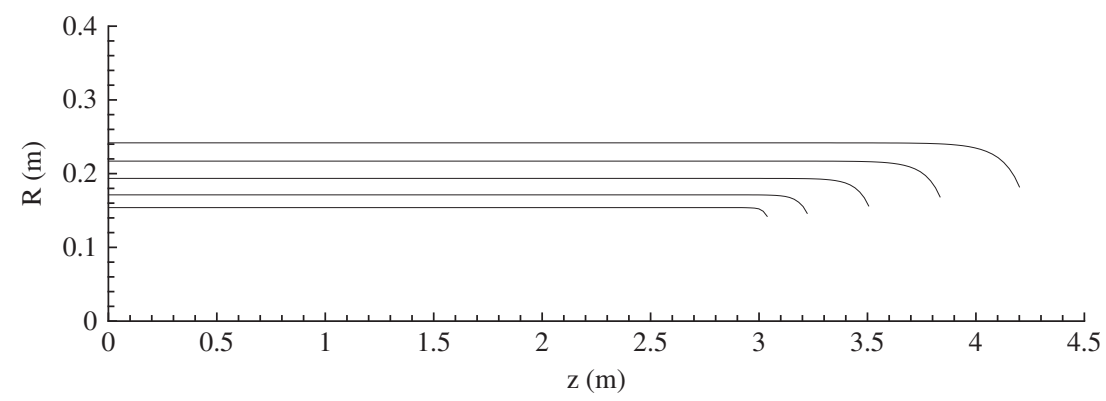

Fig. 3. Change of the geometry versus the pressure with fabric no. $1, p=20 \mathrm{kPa}, 50 \mathrm{kPa}, 100 \mathrm{kPa}, 150 \mathrm{kPa}, 200 \mathrm{kPa}$.
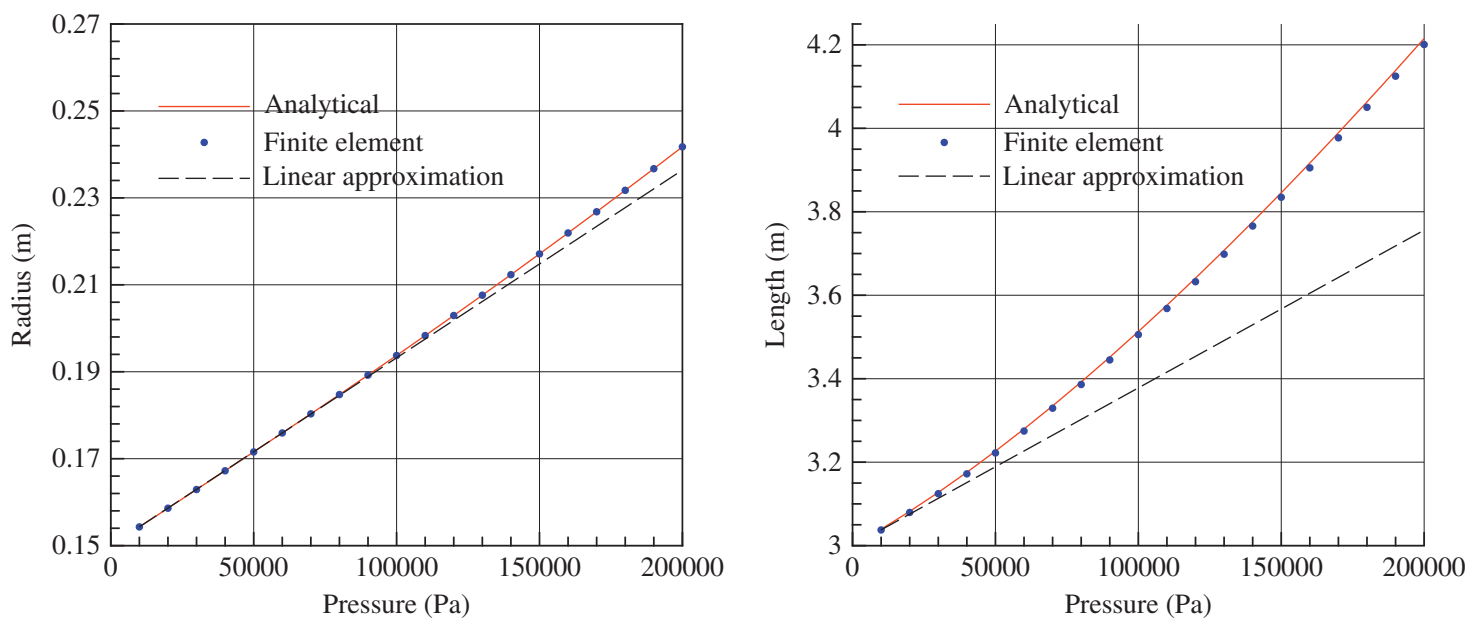

Fig. 4. Radius and length versus the pressure. (Balanced) fabric no. $1: E_{\ell}^{*}=E_{t}^{*}=50000 \mathrm{~Pa} \mathrm{~m}, G_{\ell t}^{*}=12500 \mathrm{~Pa} \mathrm{~m}, v_{\ell t}=v_{t \ell}=0.08$.
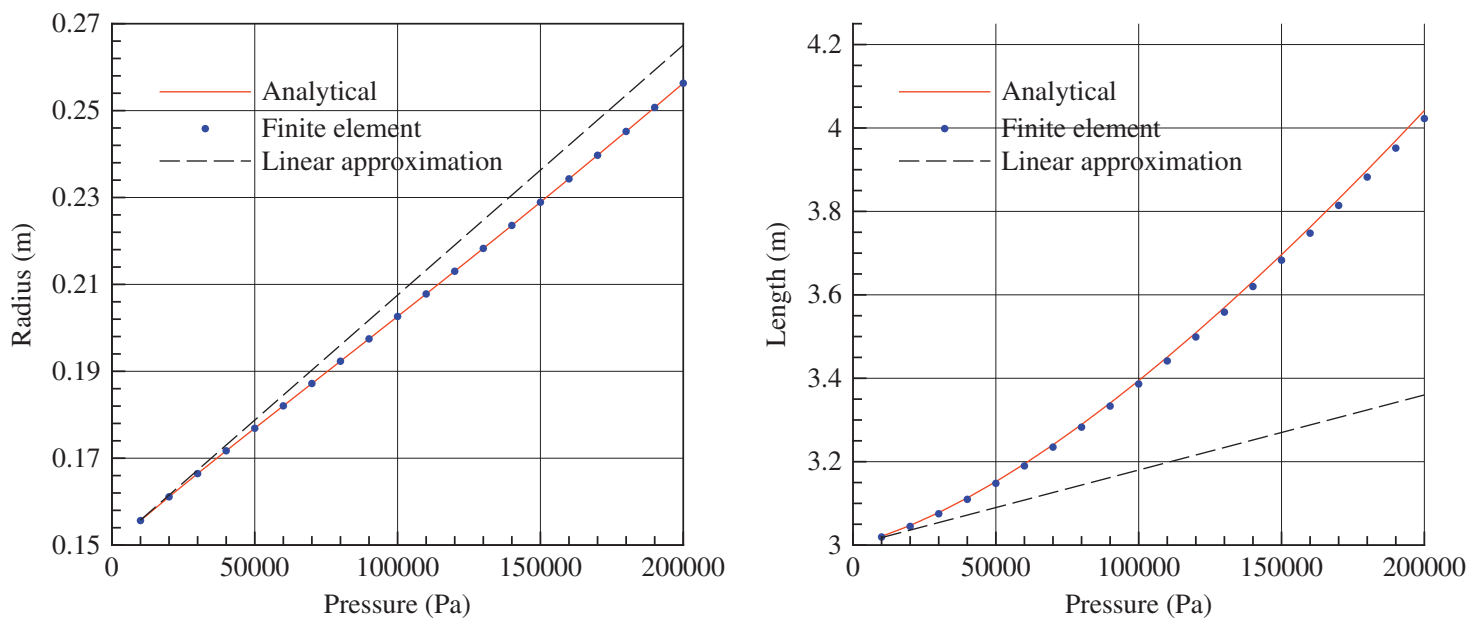

Fig. 5. Radius and length versus the pressure. (Unbalanced) fabric no. $2: E_{\ell}^{*}=50000 \mathrm{~Pa} \mathrm{~m}, E_{t}^{*}=35000 \mathrm{~Pa} \mathrm{~m}, G_{\ell t}^{*}=12500 \mathrm{~Pa} \mathrm{~m}, v_{\ell t}=0.3, v_{t \ell}=0.21$.

Figs. 4 and 5 show (i) the analytical values for the radius and the length given by Relations (14) and (16), and (ii) the values given by the finite element code, versus the internal pressure. For both materials, the final radius $a$ and particularly the final length $\ell$ are nonlinear functions of the pressure.

Table 2 shows that the analytical solutions are in very good agreement with the finite element results. At the highest pressure $p=200 \mathrm{kPa}$, the maximal difference on the radius is less than $0.01 \%$ and that on the length less than $0.5 \%$.

For comparison, the results from the linear approximation, Eq. (17), are also displayed in Figs. 4 and 5 and Table 2. It can be seen that the linear formulation deviates notably from the nonlinear one as the pressure increases. For the balanced fabric no. 1 at
Table 2

Maximal differences between analytical and finite element results.

\begin{tabular}{lcclll}
\hline & \multicolumn{3}{l}{$\begin{array}{l}\text { Linear approximation (Eq. } \\
(17))\end{array}$} & \multicolumn{2}{l}{$\begin{array}{l}\text { Nonlinear solution (Eqs. (14) } \\
\text { and (16)) }\end{array}$} \\
\cline { 2 - 3 } \cline { 5 - 6 } & Fabric no. 1 & Fabric no. 2 & & Fabric no. 1 & Fabric no. 2 \\
\hline Final radius, $a(\%)$ & 2.21 & 3.42 & & $<0.01$ & $<0.01$ \\
Final length, $\ell(\%)$ & 10.56 & 16.47 & 0.38 & 0.48 \\
\hline
\end{tabular}

$p=200 \mathrm{kPa}$, the difference with the finite element results is about $2 \%$ for the radius and more than $10 \%$ for the length. For the unbalanced fabric no. 2, the differences are more significant: 
about $3 \%$ for the radius and more than $16 \%$ for the length. This shows that the nonlinear formulation (14) and (16) agree very well with the finite element results.

\section{Conclusion}

In this paper, analytical formulae have been proposed for an orthotropic membrane tube obeying to the Saint-Venant Kirchhoff hyperelastic law, with the orthotropy axes parallel to the circumference and the axis of the tube, and inflated by an internal pressure. As regards the geometry, it has been shown that the final length and radius of the tube can be obtained by solving a simple cubic equation and that they vary as nonlinear functions of the inflating pressure. In order to assess the analytical relations, numerical examples have been carried out using different material properties and the results have been found to be in very good accordance with finite element computations.

\section{References}

[1] Comer RL, Levy S. Deflections of an inflated circular cylindrical cantilever beam. AIAA Journal 1963;1(7):1652-5.

[2] Douglass WJ. Bending stiffness of an inflated cylindrical cantilever beam. AIAA Journal 1969;7:1248-53.

[3] Webber JPH. Deflections of inflated cylindrical cantilever beams subjected to bending and torsion. Aeronautical Journal 1982;1020:306-12.
[4] Main JA, Peterson SW, Strauss AM. Load-deflection behaviour of space-based inflatable fabric beams. Journal of Aerospace Engineering 1994;7(2):225-38.

[5] Main JA, Peterson SW, Strauss AM. Beam-type bending of space-based inflated membrane structures. Journal of Aerospace Engineering 1995;8(2): 120-5.

[6] Main JA, Carlin RA, Garcia E, Peterson SW, Strauss AM. Dynamic analysis of space-based inflated beam structures. The Journal of the Acoustical Society of America 1995;97:1035-42.

[7] Suhey JD, Kim NH, Niezrecki C. Numerical modeling and design of inflatable structures-application to open-ocean-aquaculture cages. Aquacultural Engineering 2005;33(4):285-303.

[8] Fichter WB. A theory for inflated thin-wall cylindrical beams. Technical reports, NASA Technical Note, NASA TND-3466; 1966.

[9] Steeves EC. A linear analysis of the deformation of pressure stabilized beams. Technical report 75-47-AMEL, US Army Natick Laboratories; 1975.

[10] Wielgosz C, Thomas JC. Deflections of inflatable fabric panels at high pressure. Thin-Walled Structures 2002;40(6):523-36.

[11] Thomas JC, Wielgosz C. Deflections of highly inflated fabric tubes. ThinWalled Structures 2004;42(7):1049-66.

[12] Le van A, Wielgosz C. Bending and buckling of inflatable beams: some new theoretical results. Thin-Walled Structures 2005;43(8):1166-87.

[13] Thomas JC, Jiang Z, Wielgosz C. Continuous and finite element methods for the vibrations of inflatable beams. International Journal of Space Structures 2006;21:197-222.

[14] Apedo KL, Ronel S, Jacquelin E, Massenzio M, Bennani A. Theoretical analysis of inflatable beams made from orthotropic fabric. Thin-Walled Structures 2009;47(12):1507-22.

[15] Wang CG, Tan HF, Du XW, He XD. A new model for wrinkling and collapse analysis of membrane inflated beam. Acta Mechanica Sinica 2010;26: 617-23.

[16] Ogden RW. Non-linear elastic deformations. New York: Dover Publications, Inc.; 1997. 